\title{
Low energy dissociation processes of ionized cyclohexene: a theoretical insight.
}

\author{
Guy Bouchoux ${ }^{1}$, Jean-Yves Salpin ${ }^{2}$ and Manuel Yáñez ${ }^{3}$ \\ 1) Laboratoire des Mécanismes Réactionnels, UMR CNRS 7651, Ecole Polytechnique, \\ F- 91128 Palaiseau cedex, France \\ 2) Laboratoire Analyse et Environnement, UMR 8587, Institut des Sciences, Université d'Evry, boulevard F. \\ Mitterrand, 91025 Evry cedex, France \\ 3) Departamento de Quimica. Universidad Autonoma de Madrid \\ Cantoblanco 28049 Madrid (Spain)

\section{SUPPORTING INFORMATION}

B3LYP/6-31G(d) geometry (cartesian coordinates) of the different minima and transition states investigated and listed in Table 2. 


\section{Minima}

\section{1}

$\begin{array}{lrrr}\mathrm{C} & -0.001035 & 0.000043 & -0.000061 \\ \mathrm{C} & -0.002229 & 1.414457 & 0.000295 \\ \mathrm{C} & 0.001230 & 2.191124 & 1.244749 \\ \mathrm{C} & -0.238034 & 1.373724 & 2.523451 \\ \mathrm{C} & 0.522647 & 0.040522 & 2.478256 \\ \mathrm{C} & 0.133221 & -0.776314 & 1.237256 \\ \mathrm{H} & -0.130838 & -0.523743 & -0.946616 \\ \mathrm{H} & 0.021213 & 1.938446 & -0.954730 \\ \mathrm{H} & 1.003873 & 2.681544 & 1.260063 \\ \mathrm{H} & -0.671608 & 3.058089 & 1.139903 \\ \mathrm{H} & 0.069076 & 1.961089 & 3.392674 \\ \mathrm{H} & -1.314750 & 1.191690 & 2.633127 \\ \mathrm{H} & 1.604736 & 0.222502 & 2.459883 \\ \mathrm{H} & 0.320074 & -0.546396 & 3.377876 \\ \mathrm{H} & 0.783336 & -1.648176 & 1.057994 \\ \mathrm{H} & -0.864992 & -1.258471 & 1.368597\end{array}$

\section{5}

$\begin{array}{lrrr}\mathrm{C} & 0.001221 & -0.000372 & -0.000231 \\ \mathrm{C} & 0.001617 & 1.550824 & -0.000256 \\ \mathrm{C} & -0.001548 & 1.959028 & 1.488615 \\ \mathrm{C} & -0.318256 & 0.732682 & 2.236004 \\ \mathrm{C} & -0.324853 & -0.383925 & 1.380135 \\ \mathrm{C} & -0.797926 & -0.713981 & -1.122537 \\ \mathrm{H} & 1.051854 & -0.363861 & -0.128138 \\ \mathrm{H} & 0.845752 & 1.969637 & -0.552520 \\ \mathrm{H} & -0.910741 & 1.912058 & -0.489997 \\ \mathrm{H} & -0.684305 & 2.785312 & 1.751060 \\ \mathrm{H} & 0.980877 & 2.339152 & 1.839887 \\ \mathrm{H} & -0.508553 & 0.689910 & 3.305734 \\ \mathrm{H} & -0.515860 & -1.404127 & 1.705543 \\ \mathrm{H} & -1.855904 & -0.441876 & -1.059990 \\ \mathrm{H} & -0.704814 & -1.800673 & -1.048073 \\ \mathrm{H} & -0.410369 & -0.399282 & -2.094320\end{array}$

\section{6(chair)}

$\begin{array}{lrrr}\mathrm{C} & -1.141020 & -0.393341 & -0.667399 \\ \mathrm{C} & -1.195317 & -0.477211 & 0.783383 \\ \mathrm{C} & 0.386147 & -0.685264 & 1.285129 \\ \mathrm{C} & 1.141064 & 0.393263 & 0.667367 \\ \mathrm{C} & 1.195305 & 0.477256 & -0.783406 \\ \mathrm{C} & -0.386205 & 0.685295 & -1.285084 \\ \mathrm{H} & 0.373113 & -0.666748 & 2.375912 \\ \mathrm{H} & -1.534040 & 0.447837 & 1.259287 \\ \mathrm{H} & -1.760340 & -1.326354 & 1.170735 \\ \mathrm{H} & -1.439535 & -1.252235 & -1.266879 \\ \mathrm{H} & 1.439887 & 1.252031 & 1.266878 \\ \mathrm{H} & 1.760415 & 1.326400 & -1.170609 \\ \mathrm{H} & 1.533914 & -0.447745 & -1.259517 \\ \mathrm{H} & -0.677763 & 1.678078 & -0.929687 \\ \mathrm{H} & -0.373019 & 0.666895 & -2.375874 \\ \mathrm{H} & 0.677529 & -1.678147 & 0.929817\end{array}$




\section{6(planar)}

$\begin{array}{lrrr}\mathrm{C} & -1.299615 & 0.042428 & -0.774119 \\ \mathrm{C} & -1.248154 & -0.000612 & 0.690816 \\ \mathrm{C} & -0.034122 & -0.043099 & 1.512342 \\ \mathrm{C} & 1.292878 & -0.196152 & 0.761721 \\ \mathrm{C} & 1.248151 & 0.000581 & -0.690841 \\ \mathrm{C} & 0.040882 & 0.196829 & -1.499926 \\ \mathrm{H} & -2.200733 & -0.001137 & 1.217998 \\ \mathrm{H} & -0.148367 & -0.820964 & 2.285359 \\ \mathrm{H} & -0.016945 & 0.887512 & 2.112290 \\ \mathrm{H} & 1.705620 & -1.208379 & 0.938469 \\ \mathrm{H} & 0.109628 & 1.209398 & -1.942931 \\ \mathrm{H} & 0.091926 & -0.454134 & -2.388363 \\ \mathrm{H} & -2.016008 & 0.819756 & -1.087541 \\ \mathrm{H} & -1.798184 & -0.888476 & -1.107533 \\ \mathrm{H} & 2.072218 & 0.455459 & 1.190336 \\ \mathrm{H} & 2.200719 & 0.001113 & -1.218040\end{array}$

\section{7}

$\begin{array}{lrrr}\mathrm{C} & 0.002109 & -0.019113 & 0.010984 \\ \mathrm{C} & 0.025232 & 1.524191 & 0.012258 \\ \mathrm{C} & -0.079935 & 2.117497 & 1.357288 \\ \mathrm{C} & -0.611589 & 1.346265 & 2.478839 \\ \mathrm{C} & -1.196783 & 0.029774 & 2.235838 \\ \mathrm{C} & -1.105376 & -0.607384 & 0.909882 \\ \mathrm{H} & 0.276170 & 3.125872 & 1.552437 \\ \mathrm{H} & -1.156976 & 1.950392 & 3.220312 \\ \mathrm{H} & 0.318435 & 1.093745 & 3.062336 \\ \mathrm{H} & -1.662168 & -0.486285 & 3.071785 \\ \mathrm{H} & -1.015979 & -1.697089 & 1.006816 \\ \mathrm{H} & -2.097226 & -0.460899 & 0.433705 \\ \mathrm{H} & 0.974045 & -0.389491 & 0.355143 \\ \mathrm{H} & -0.126468 & -0.381396 & -1.011456 \\ \mathrm{H} & 0.898158 & 1.919925 & -0.522201 \\ \mathrm{H} & -0.841930 & 1.914591 & -0.560110\end{array}$

\section{8}

$\begin{array}{lrrr}\mathrm{C} & -0.019656 & 0.010254 & 0.083050 \\ \mathrm{C} & 1.449636 & 0.060636 & 0.312041 \\ \mathrm{C} & 2.199153 & 1.317167 & 0.055256 \\ \mathrm{C} & 1.468443 & 2.125871 & -1.043570 \\ \mathrm{C} & 0.904121 & 1.103452 & -2.058447 \\ \mathrm{C} & 0.444998 & -0.104717 & -1.325698 \\ \mathrm{H} & 1.961120 & -0.844733 & 0.631941 \\ \mathrm{H} & 0.475992 & -1.089031 & -1.788138 \\ \mathrm{H} & 1.645512 & 0.818571 & -2.815221 \\ \mathrm{H} & 0.663475 & 2.728447 & -0.611307 \\ \mathrm{H} & 3.233985 & 1.081935 & -0.222852 \\ \mathrm{H} & -0.535202 & 0.949965 & 0.304969 \\ \mathrm{H} & -0.517741 & -0.873154 & 0.477762 \\ \mathrm{H} & 0.049387 & 1.516813 & -2.622713 \\ \mathrm{H} & 2.151932 & 2.823715 & -1.532817 \\ \mathrm{H} & 2.268655 & 1.883393 & 1.001016\end{array}$


9

$\begin{array}{lrrr}\mathrm{C} & -0.960017 & 0.627929 & -1.090928 \\ \mathrm{C} & -0.956817 & 0.817688 & 0.562615 \\ \mathrm{C} & 0.445613 & 0.670407 & 1.176920 \\ \mathrm{C} & 1.363373 & -0.278978 & 0.363016 \\ \mathrm{C} & 0.516210 & -1.367954 & -0.372817 \\ \mathrm{C} & -0.642494 & -0.724301 & -0.932946 \\ \mathrm{H} & 0.219609 & -2.213791 & 0.264973 \\ \mathrm{H} & 1.920982 & 0.286405 & -0.389644 \\ \mathrm{H} & 0.326793 & 0.294116 & 2.197494 \\ \mathrm{H} & -0.164411 & 1.271644 & -1.489118 \\ \mathrm{H} & -1.941103 & 0.865940 & -1.507219 \\ \mathrm{H} & 1.082916 & -1.817308 & -1.209585 \\ \mathrm{H} & 2.098627 & -0.778204 & 0.999431 \\ \mathrm{H} & 0.926773 & 1.649810 & 1.251113 \\ \mathrm{H} & -1.373848 & 1.828533 & 0.634395 \\ \mathrm{H} & -1.691547 & 0.144109 & 1.012998\end{array}$

\section{0}

$\begin{array}{lrrr}\mathrm{C} & -1.473986 & -0.041669 & 0.060888 \\ \mathrm{C} & -1.154223 & 0.856782 & 1.050466 \\ \mathrm{C} & 1.099149 & -0.880531 & 1.088427 \\ \mathrm{C} & 1.469582 & 0.038598 & 0.136140 \\ \mathrm{C} & 0.726899 & 0.327102 & -1.127430 \\ \mathrm{C} & -0.668348 & -0.300882 & -1.170081 \\ \mathrm{H} & -2.371669 & -0.646114 & 0.191558 \\ \mathrm{H} & -0.402037 & 1.629178 & 0.921019 \\ \mathrm{H} & 0.354504 & -1.649013 & 0.903752 \\ \mathrm{H} & 2.360503 & 0.637687 & 0.324881 \\ \mathrm{H} & 1.334866 & -0.013187 & -1.981103 \\ \mathrm{H} & -1.232528 & 0.060453 & -2.044913 \\ \mathrm{H} & 0.678134 & 1.418237 & -1.251372 \\ \mathrm{H} & -0.612933 & -1.388734 & -1.317516 \\ \mathrm{H} & -1.717784 & 0.884056 & 1.979251 \\ \mathrm{H} & 1.614498 & -0.928962 & 2.043976\end{array}$

\section{1}

$\begin{array}{lrrr}\mathrm{C} & -1.347915 & -0.243078 & -0.739531 \\ \mathrm{C} & -1.245915 & -0.440939 & 0.710685 \\ \mathrm{C} & -0.077443 & 0.188607 & 1.280442 \\ \mathrm{C} & 0.910367 & 0.479222 & 0.028985 \\ \mathrm{C} & -0.132287 & 0.615538 & -1.139311 \\ \mathrm{H} & -2.321775 & 0.180882 & -1.036249 \\ \mathrm{H} & 0.288654 & 0.326959 & -2.104599 \\ \mathrm{H} & -0.417639 & 1.669585 & -1.210928 \\ \mathrm{H} & -1.358120 & -1.244829 & -1.209363 \\ \mathrm{C} & 1.866206 & -0.614498 & -0.061537 \\ \mathrm{H} & 1.542555 & -1.634581 & -0.259063 \\ \mathrm{H} & 2.927941 & -0.458851 & 0.104756 \\ \mathrm{H} & -0.346816 & 1.215503 & 1.594586 \\ \mathrm{H} & 0.336494 & -0.304146 & 2.168395 \\ \mathrm{H} & -1.919941 & -1.082773 & 1.275049 \\ \mathrm{H} & 1.430564 & 1.423136 & 0.199016\end{array}$


12

$\begin{array}{lrrr}\mathrm{C} & 0.221305 & -0.243384 & -0.433963 \\ \mathrm{C} & 0.232004 & 0.070878 & 1.033307 \\ \mathrm{C} & 1.632882 & 0.114249 & 1.536735 \\ \mathrm{C} & 2.507608 & 0.014077 & 0.357232 \\ \mathrm{C} & 1.689851 & 0.008525 & -0.868850 \\ \mathrm{H} & -0.487286 & 0.367548 & -1.003590 \\ \mathrm{H} & 1.901561 & -0.714595 & 2.228933 \\ \mathrm{H} & -0.640597 & 0.126080 & 1.670291 \\ \mathrm{H} & 1.916699 & 1.003725 & 2.134771 \\ \mathrm{H} & 2.096300 & -0.618939 & -1.671779 \\ \mathrm{H} & 1.811334 & 1.048756 & -1.246497 \\ \mathrm{H} & -0.061754 & -1.292431 & -0.621427 \\ \mathrm{C} & 3.968146 & -0.074323 & 0.392561 \\ \mathrm{H} & 4.404792 & 0.251279 & 1.339761 \\ \mathrm{H} & 4.222289 & -1.143635 & 0.256434 \\ \mathrm{H} & 4.430466 & 0.437072 & -0.460604\end{array}$

\section{3}

$\begin{array}{lrrr}\mathrm{C} & -0.950393 & 0.636574 & -1.264118 \\ \mathrm{C} & -0.952525 & 0.371615 & 0.559980 \\ \mathrm{C} & 0.327150 & 0.665914 & 1.236532 \\ \mathrm{C} & 1.429801 & 0.006893 & 0.375101 \\ \mathrm{C} & 0.703543 & -1.122878 & -0.394097 \\ \mathrm{C} & -0.742734 & -0.629800 & -0.571042 \\ \mathrm{H} & -1.926282 & 0.673109 & 0.937467 \\ \mathrm{H} & -1.559667 & -1.346451 & -0.687154 \\ \mathrm{H} & 0.672458 & -2.044766 & 0.195131 \\ \mathrm{H} & 1.864141 & 0.742395 & -0.310198 \\ \mathrm{H} & 0.248258 & 0.177319 & 2.228801 \\ \mathrm{H} & -0.124699 & 1.297406 & -1.515508 \\ \mathrm{H} & -1.941446 & 0.930310 & -1.599365 \\ \mathrm{H} & 1.166006 & -1.354031 & -1.357579 \\ \mathrm{H} & 2.250850 & -0.377065 & 0.983348 \\ \mathrm{H} & 0.461328 & 1.731862 & 1.470918\end{array}$

\section{4}

$\begin{array}{lrrr}\mathrm{C} & -0.396965 & 0.369173 & -0.187729 \\ \mathrm{C} & 1.119645 & 0.377227 & 0.117707 \\ \mathrm{C} & 1.519040 & 1.791906 & 0.107158 \\ \mathrm{C} & 0.395825 & 2.654667 & 0.029365 \\ \mathrm{C} & -0.844403 & 1.835871 & 0.012391 \\ \mathrm{H} & -0.948017 & -0.325851 & 0.448188 \\ \mathrm{H} & 1.740269 & -0.231397 & -0.559585 \\ \mathrm{H} & 1.357129 & -0.043276 & 1.117354 \\ \mathrm{H} & 2.546438 & 2.143662 & 0.168253 \\ \mathrm{H} & -1.575782 & 2.206612 & -0.720483 \\ \mathrm{H} & -1.348754 & 1.983671 & 0.987755 \\ \mathrm{H} & -0.566677 & 0.062738 & -1.224944 \\ \mathrm{C} & 0.445186 & 4.126220 & -0.033176 \\ \mathrm{H} & 1.398363 & 4.537274 & 0.308708 \\ \mathrm{H} & 0.311201 & 4.432663 & -1.086799 \\ \mathrm{H} & -0.389568 & 4.581771 & 0.512329\end{array}$


15

$\begin{array}{lrrr}\mathrm{C} & -0.669800 & -0.313910 & -0.390516 \\ \mathrm{C} & -0.579677 & -0.065905 & 1.065100 \\ \mathrm{C} & 0.593145 & 0.072161 & -1.057259 \\ \mathrm{C} & -1.803076 & -0.844895 & -1.051314 \\ \mathrm{H} & -1.505232 & 0.314919 & 1.515591 \\ \mathrm{H} & 0.897499 & -0.599702 & -1.870010 \\ \mathrm{H} & -2.668159 & -1.190754 & -0.490362 \\ \mathrm{H} & -1.835885 & -0.919604 & -2.135746 \\ \mathrm{C} & 0.707381 & 0.764935 & 1.260467 \\ \mathrm{C} & 1.601964 & 0.317242 & 0.086004 \\ \mathrm{H} & 1.163219 & 0.598363 & 2.238167 \\ \mathrm{H} & 2.361681 & 1.053405 & -0.182948 \\ \mathrm{H} & 2.117379 & -0.620964 & 0.322396 \\ \mathrm{H} & 0.465887 & 1.831501 & 1.183821 \\ \mathrm{H} & -0.448373 & -1.079609 & 1.508437 \\ \mathrm{H} & 0.352357 & 1.034673 & -1.564233\end{array}$

\begin{tabular}{rrrr}
\hline 16 & & & \\
$\mathrm{C}$ & -1.281344 & -0.137048 & -0.776587 \\
$\mathrm{C}$ & -1.387903 & -0.133377 & 0.787436 \\
$\mathrm{C}$ & -0.036765 & 0.170921 & 1.185214 \\
$\mathrm{C}$ & 0.912379 & 0.489294 & 0.172246 \\
$\mathrm{C}$ & -0.013285 & 0.713859 & -1.071899 \\
$\mathrm{H}$ & -2.183400 & 0.271514 & -1.235750 \\
$\mathrm{H}$ & -1.852175 & -0.990446 & 1.290633 \\
$\mathrm{H}$ & -1.954770 & 0.753776 & 1.151324 \\
$\mathrm{H}$ & 0.491573 & 0.424904 & -1.997282 \\
$\mathrm{H}$ & -0.259829 & 1.778186 & -1.135643 \\
$\mathrm{H}$ & -1.164320 & -1.168534 & -1.120259 \\
$\mathrm{C}$ & 1.724175 & -0.897128 & 0.109978 \\
$\mathrm{H}$ & 2.335975 & -1.026847 & 1.003062 \\
$\mathrm{H}$ & 1.080255 & -1.767329 & -0.034209 \\
$\mathrm{H}$ & 2.364439 & -0.785675 & -0.770732 \\
$\mathrm{H}$ & 1.638706 & 1.271319 & 0.410521 \\
& & & \\
\hline
\end{tabular}

\section{C5H7+}

$\begin{array}{lrrr}\mathrm{C} & -0.102688 & -2.490781 & 0.098451 \\ \mathrm{C} & 0.020506 & -0.959815 & 0.082912 \\ \mathrm{C} & -0.009882 & -0.585566 & 1.510588 \\ \mathrm{C} & -0.134474 & -1.694902 & 2.341074 \\ \mathrm{C} & -0.190554 & -2.826285 & 1.533307 \\ \mathrm{H} & 0.749885 & -3.010189 & -0.367870 \\ \mathrm{H} & -0.790316 & -0.454203 & -0.465811 \\ \mathrm{H} & 0.944183 & -0.592416 & -0.392536 \\ \mathrm{H} & 0.058248 & 0.442301 & 1.860732 \\ \mathrm{H} & -0.178679 & -1.680482 & 3.423605 \\ \mathrm{H} & -0.287813 & -3.844455 & 1.904288 \\ \mathrm{H} & -0.984625 & -2.871635 & -0.441321\end{array}$

\section{CH3}

$\begin{array}{lrrr}\mathrm{C} & 0.000000 & 1.565514 & -3.173869 \\ \mathrm{H} & 0.937244 & 2.075849 & -2.991956 \\ \mathrm{H} & -0.937244 & 2.075849 & -2.991956 \\ \mathrm{H} & 0.000000 & 0.546142 & -3.538701\end{array}$




\section{$\mathrm{C}^{4} \mathrm{H6}^{+}$}

$\begin{array}{lrrr}\mathrm{C} & 0.008346 & 0.000000 & -0.011334 \\ \mathrm{C} & 0.003374 & 0.000000 & 1.378336 \\ \mathrm{C} & 1.200767 & 0.000000 & 2.124139 \\ \mathrm{C} & 1.195800 & 0.000000 & 3.513810 \\ \mathrm{H} & 0.939548 & 0.000000 & -0.573633 \\ \mathrm{H} & -0.918698 & 0.000000 & -0.578150 \\ \mathrm{H} & -0.948528 & 0.000000 & 1.906002 \\ \mathrm{H} & 2.152667 & 0.000000 & 1.596471 \\ \mathrm{H} & 2.122849 & 0.000000 & 4.080619 \\ \mathrm{H} & 0.264603 & 0.000000 & 4.076114\end{array}$

\section{C2H4}

$\begin{array}{lrrr}\mathrm{C} & 0.000000 & 0.000000 & 0.001954 \\ \mathrm{C} & 0.000000 & 0.000000 & 1.332946 \\ \mathrm{H} & 0.923822 & 0.000000 & -0.571867 \\ \mathrm{H} & -0.923822 & 0.000000 & -0.571867 \\ \mathrm{H} & 0.923823 & 0.000000 & 1.906767 \\ \mathrm{H} & -0.923823 & 0.000000 & 1.906767\end{array}$




\section{Transition states}

\section{TS1/6}

\begin{tabular}{lrrr}
$\mathrm{C}$ & -1.102319 & 0.663597 & -0.818846 \\
$\mathrm{C}$ & -1.132663 & 0.026381 & 0.588341 \\
$\mathrm{C}$ & 0.049078 & 0.284237 & 1.409011 \\
$\mathrm{C}$ & 1.321300 & 0.303273 & 0.619743 \\
$\mathrm{C}$ & 1.118239 & -0.587997 & -0.657247 \\
$\mathrm{C}$ & -0.332922 & -0.584654 & -1.045382 \\
$\mathrm{H}$ & -2.074262 & 0.716798 & -1.304962 \\
$\mathrm{H}$ & 0.008173 & 0.211213 & 2.489589 \\
$\mathrm{H}$ & 2.174035 & -0.047711 & 1.207243 \\
$\mathrm{H}$ & -0.770246 & -1.389856 & -1.637667 \\
$\mathrm{H}$ & 1.567188 & 1.331805 & 0.315684 \\
$\mathrm{H}$ & -0.549543 & 1.598821 & -0.876957 \\
$\mathrm{H}$ & 1.682395 & -0.173017 & -1.503766 \\
$\mathrm{H}$ & 1.472430 & -1.611571 & -0.504989 \\
$\mathrm{H}$ & -2.128829 & -0.127641 & 1.009407 \\
$\mathrm{H}$ & -0.905620 & -1.137863 & 0.232697 \\
\hline
\end{tabular}

\section{TS1/7}

$\begin{array}{lrrr}\text { C } & 0.000000 & 0.000000 & 0.000000 \\ \mathrm{C} & 0.000000 & 1.485743 & 0.000000 \\ \mathrm{C} & 0.000000 & 2.219005 & 1.243249 \\ \mathrm{C} & -0.193555 & 1.538189 & 2.500654 \\ \mathrm{C} & -0.200054 & 0.060130 & 2.564641 \\ \mathrm{C} & 0.458733 & -0.608276 & 1.339210 \\ \mathrm{H} & -0.008353 & 2.044946 & -0.930229 \\ \mathrm{H} & -0.169463 & 3.296325 & 1.217767 \\ \mathrm{H} & 1.076478 & 2.106363 & 1.736184 \\ \mathrm{H} & -0.256830 & 2.143702 & 3.402807 \\ \mathrm{H} & 0.227974 & -0.296362 & 3.507922 \\ \mathrm{H} & -1.274785 & -0.205618 & 2.615317 \\ \mathrm{H} & 1.547359 & -0.505466 & 1.430345 \\ \mathrm{H} & 0.246400 & -1.679647 & 1.356068 \\ \mathrm{H} & 0.608272 & -0.380627 & -0.830108 \\ \mathrm{H} & -1.029593 & -0.326712 & -0.237986\end{array}$

\begin{tabular}{|c|c|c|c|}
\hline \multicolumn{4}{|c|}{ TS5/12 } \\
\hline C & -0.398241 & 0.150995 & -0.094784 \\
\hline C & -0.154646 & 1.635112 & -0.060139 \\
\hline $\mathrm{C}$ & -0.033146 & 2.024413 & 1.443640 \\
\hline C & -0.375282 & 0.771221 & 2.190479 \\
\hline $\mathrm{C}$ & -0.469690 & -0.329698 & 1.261439 \\
\hline C & -0.576504 & -0.657874 & -1.323623 \\
\hline $\mathrm{H}$ & 0.644908 & -0.484936 & 0.802608 \\
\hline $\mathrm{H}$ & 0.695396 & 1.938193 & -0.681274 \\
\hline $\mathrm{H}$ & -1.040731 & 2.092770 & -0.528604 \\
\hline $\mathrm{H}$ & -0.695979 & 2.855197 & 1.715239 \\
\hline $\mathrm{H}$ & 0.982769 & 2.364773 & 1.696901 \\
\hline $\mathrm{H}$ & -0.453490 & 0.672631 & 3.265512 \\
\hline $\mathrm{H}$ & -0.816903 & -1.331077 & 1.513009 \\
\hline $\mathrm{H}$ & -1.549553 & -0.378954 & -1.758083 \\
\hline $\mathrm{H}$ & -0.594947 & -1.732431 & -1.126637 \\
\hline $\mathrm{H}$ & 0.179949 & -0.424716 & -2.080727 \\
\hline
\end{tabular}




\section{TS6/7}

$\begin{array}{lrrr}\text { C } & -1.257874 & 0.315718 & -0.735871 \\ \mathrm{C} & -1.067781 & 0.617279 & 0.718641 \\ \mathrm{C} & 0.157477 & 0.424067 & 1.373883 \\ \mathrm{C} & 1.373079 & -0.077405 & 0.664672 \\ \mathrm{C} & 1.148911 & -0.491525 & -0.752226 \\ \mathrm{C} & -0.227947 & -0.690885 & -1.296633 \\ \mathrm{H} & 0.242718 & 0.733685 & 2.416011 \\ \mathrm{H} & 1.858360 & -0.859836 & 1.272845 \\ \mathrm{H} & 2.082451 & 0.770030 & 0.727982 \\ \mathrm{H} & 2.014199 & -0.784133 & -1.335748 \\ \mathrm{H} & -0.222675 & -0.630025 & -2.389149 \\ \mathrm{H} & -0.587239 & -1.710433 & -1.064866 \\ \mathrm{H} & -1.155402 & 1.291361 & -1.237068 \\ \mathrm{H} & -2.287115 & -0.009009 & -0.919083 \\ \mathrm{H} & -1.877029 & 1.088122 & 1.278946 \\ \mathrm{H} & -0.823473 & -0.473239 & 1.415336\end{array}$

\section{TS6/10}

$\begin{array}{lrrr}\mathrm{C} & -0.985188 & -0.746877 & -0.691791 \\ \mathrm{C} & -1.159460 & -0.839208 & 0.687160 \\ \mathrm{C} & 0.976866 & -0.772018 & 0.983361 \\ \mathrm{C} & 0.972225 & 0.612857 & 0.830218 \\ \mathrm{C} & 0.527527 & 1.302258 & -0.415559 \\ \mathrm{C} & -0.335029 & 0.414894 & -1.364585 \\ \mathrm{H} & -1.168987 & -1.635871 & -1.294135 \\ \mathrm{H} & -1.375877 & 0.041456 & 1.286070 \\ \mathrm{H} & 1.219327 & -1.428110 & 0.151558 \\ \mathrm{H} & 1.129284 & 1.229017 & 1.714835 \\ \mathrm{H} & 1.407585 & 1.663893 & -0.970146 \\ \mathrm{H} & -1.105489 & 1.044702 & -1.836379 \\ \mathrm{H} & -0.008808 & 2.212860 & -0.123471 \\ \mathrm{H} & 0.259592 & 0.030463 & -2.201583 \\ \mathrm{H} & -1.471904 & -1.781277 & 1.132854 \\ \mathrm{H} & 1.133640 & -1.208573 & 1.967568\end{array}$

\section{TS7/8}

$\begin{array}{lrrr}\mathrm{C} & 0.000000 & 0.000000 & 0.000000 \\ \mathrm{C} & 0.000000 & 0.000000 & 1.470987 \\ \mathrm{C} & 1.425664 & 0.000000 & -0.494876 \\ \mathrm{C} & 2.463120 & 0.832021 & 0.178987 \\ \mathrm{C} & 1.851458 & 1.659565 & 1.332364 \\ \mathrm{C} & 0.980501 & 0.775024 & 2.211936 \\ \mathrm{H} & -0.495224 & 0.976304 & -0.247548 \\ \mathrm{H} & -0.630534 & -0.780482 & -0.430734 \\ \mathrm{H} & -0.720490 & -0.601358 & 2.027876 \\ \mathrm{H} & 1.664659 & -0.675231 & -1.311754 \\ \mathrm{H} & 2.950253 & 1.503457 & -0.545447 \\ \mathrm{H} & 3.268302 & 0.185399 & 0.569412 \\ \mathrm{H} & 2.632053 & 2.112690 & 1.948538 \\ \mathrm{H} & 1.248132 & 2.481840 & 0.930247 \\ \mathrm{H} & 1.556741 & -0.026791 & 2.735792 \\ \mathrm{H} & 0.488514 & 1.268562 & 3.068067\end{array}$




\section{TS8/9}

$\begin{array}{lrrr}\mathrm{C} & -0.946563 & 0.677055 & -1.103793 \\ \mathrm{C} & -0.988353 & 0.570558 & 0.458149 \\ \mathrm{C} & 0.337123 & 0.699354 & 1.192223 \\ \mathrm{C} & 1.387001 & -0.130651 & 0.398040 \\ \mathrm{C} & 0.656524 & -1.259180 & -0.415494 \\ \mathrm{C} & -0.676590 & -0.696231 & -0.756748 \\ \mathrm{H} & -1.931279 & 0.933335 & 0.874871 \\ \mathrm{H} & 0.557358 & -2.189472 & 0.152669 \\ \mathrm{H} & 1.923820 & 0.513788 & -0.302594 \\ \mathrm{H} & 0.218754 & 0.339831 & 2.218868 \\ \mathrm{H} & -0.124170 & 1.254832 & -1.524741 \\ \mathrm{H} & -1.916943 & 0.912918 & -1.542118 \\ \mathrm{H} & 1.185280 & -1.489441 & -1.347545 \\ \mathrm{H} & 2.128979 & -0.580340 & 1.061535 \\ \mathrm{H} & 0.625879 & 1.754031 & 1.248165 \\ \mathrm{H} & -1.282523 & -0.614909 & 0.526626\end{array}$

\section{TS8/13}

$\begin{array}{lrrr}\mathrm{C} & -0.737346 & 0.712559 & -1.333746 \\ \mathrm{C} & -0.788753 & 0.735798 & 0.453852 \\ \mathrm{C} & 0.516881 & 0.701972 & 1.143477 \\ \mathrm{C} & 1.369303 & -0.361917 & 0.415351 \\ \mathrm{C} & 0.338330 & -1.329310 & -0.213204 \\ \mathrm{C} & -0.893683 & -0.476898 & -0.537493 \\ \mathrm{H} & -1.640112 & 1.323910 & 0.785046 \\ \mathrm{H} & -1.890103 & -0.923935 & -0.540631 \\ \mathrm{H} & 0.039232 & -2.103985 & 0.499205 \\ \mathrm{H} & 1.994563 & 0.110498 & -0.349751 \\ \mathrm{H} & 0.302726 & 0.399460 & 2.187299 \\ \mathrm{H} & 0.240915 & 1.073787 & -1.642964 \\ \mathrm{H} & -1.604056 & 1.232817 & -1.732521 \\ \mathrm{H} & 0.712641 & -1.826752 & -1.113592 \\ \mathrm{H} & 2.047010 & -0.880250 & 1.096354 \\ \mathrm{H} & 0.968794 & 1.701229 & 1.242131\end{array}$

\section{TS9/15}

$\begin{array}{lrrr}\mathrm{C} & -1.109970 & 0.407005 & -1.018091 \\ \mathrm{C} & -1.046930 & 0.858568 & 0.561204 \\ \mathrm{C} & 0.268595 & 0.501988 & 1.289054 \\ \mathrm{C} & 1.374487 & -0.014505 & 0.351042 \\ \mathrm{C} & 0.810872 & -1.207954 & -0.491657 \\ \mathrm{C} & -0.488677 & -0.856335 & -0.973487 \\ \mathrm{H} & 0.780596 & -2.166508 & 0.050187 \\ \mathrm{H} & 1.717883 & 0.771968 & -0.329364 \\ \mathrm{H} & 0.065657 & -0.262147 & 2.047102 \\ \mathrm{H} & -0.476867 & 1.124850 & -1.566850 \\ \mathrm{H} & -2.135828 & 0.434196 & -1.393788 \\ \mathrm{H} & 1.439662 & -1.410522 & -1.383113 \\ \mathrm{H} & 2.244925 & -0.366265 & 0.911584 \\ \mathrm{H} & 0.636752 & 1.384232 & 1.822109 \\ \mathrm{H} & -1.205435 & 1.937811 & 0.483888 \\ \mathrm{H} & -1.917609 & 0.419779 & 1.049858\end{array}$




\section{TS11/12}

$\begin{array}{lrrr}\mathrm{C} & -1.328905 & 0.453818 & -0.826265 \\ \mathrm{C} & -1.317789 & 0.637160 & 0.659702 \\ \mathrm{C} & 0.002809 & 0.307323 & 1.265055 \\ \mathrm{C} & 0.802290 & -0.239286 & 0.077664 \\ \mathrm{C} & 0.164624 & 0.311661 & -1.216415 \\ \mathrm{H} & -1.793435 & 1.298226 & -1.349366 \\ \mathrm{H} & 0.342383 & -0.334130 & -2.079397 \\ \mathrm{H} & 0.584993 & 1.296942 & -1.438514 \\ \mathrm{H} & -1.919677 & -0.433281 & -1.109956 \\ \mathrm{C} & 1.486622 & -1.472856 & 0.125798 \\ \mathrm{H} & 1.717974 & -1.954737 & 1.075523 \\ \mathrm{H} & 1.940677 & -1.887231 & -0.773574 \\ \mathrm{H} & 0.483823 & 1.218199 & 1.666411 \\ \mathrm{H} & -0.045038 & -0.394817 & 2.108102 \\ \mathrm{H} & -2.110135 & 1.119172 & 1.220825 \\ \mathrm{H} & 1.940529 & 0.084743 & 0.166714\end{array}$

\section{TS11/13}

$\begin{array}{lrrr}\mathrm{C} & -0.928287 & 0.522080 & -1.677533 \\ \mathrm{C} & -0.671833 & 0.740629 & 0.709582 \\ \mathrm{C} & 0.665336 & 0.712589 & 1.139579 \\ \mathrm{C} & 1.421091 & -0.386980 & 0.476425 \\ \mathrm{C} & 0.338169 & -1.222882 & -0.252221 \\ \mathrm{C} & -0.895731 & -0.278747 & -0.416518 \\ \mathrm{H} & -1.356715 & 1.552528 & 0.950551 \\ \mathrm{H} & -1.841679 & -0.809868 & -0.283221 \\ \mathrm{H} & 0.058871 & -2.080491 & 0.369022 \\ \mathrm{H} & 2.102228 & 0.127040 & -0.224399 \\ \mathrm{H} & -0.488380 & 0.132834 & 1.777993 \\ \mathrm{H} & -0.015889 & 0.937972 & -2.094827 \\ \mathrm{H} & -1.868178 & 0.756565 & -2.163921 \\ \mathrm{H} & 0.686528 & -1.611654 & -1.210437 \\ \mathrm{H} & 2.065572 & -0.952590 & 1.156616 \\ \mathrm{H} & 1.085166 & 1.427529 & 1.846739\end{array}$

\section{TS11/15}

$\begin{array}{lrrr}\mathrm{C} & -0.811461 & 0.638648 & -1.409051 \\ \mathrm{C} & -0.671820 & 0.855867 & 0.468024 \\ \mathrm{C} & 0.648997 & 0.707605 & 1.090883 \\ \mathrm{C} & 1.379053 & -0.484814 & 0.572763 \\ \mathrm{C} & 0.311907 & -1.348527 & -0.149711 \\ \mathrm{C} & -0.891623 & -0.425959 & -0.415410 \\ \mathrm{H} & -0.583824 & 1.754958 & -0.314713 \\ \mathrm{H} & -1.879198 & -0.867803 & -0.304308 \\ \mathrm{H} & -0.024595 & -2.162699 & 0.496975 \\ \mathrm{H} & 2.187667 & -0.170411 & -0.108640 \\ \mathrm{H} & 0.133312 & 0.874503 & -1.897232 \\ \mathrm{H} & -1.717570 & 1.071521 & -1.830362 \\ \mathrm{H} & 0.684357 & -1.801523 & -1.072277 \\ \mathrm{H} & 1.888408 & -1.031372 & 1.377236 \\ \mathrm{H} & -1.563858 & 1.186935 & 1.004246 \\ \mathrm{H} & 1.084982 & 1.488966 & 1.704077\end{array}$




\section{TS12/14}

$\begin{array}{lrrr}\text { C } & 0.000000 & 0.000000 & 0.000000 \\ \mathrm{C} & 0.000000 & 0.000000 & 1.561252 \\ \mathrm{C} & 1.439334 & 0.000000 & 1.955049 \\ \mathrm{C} & 2.269453 & -0.005832 & 0.764822 \\ \mathrm{C} & 1.444852 & -0.103235 & -0.416306 \\ \mathrm{C} & 1.920764 & -0.238355 & -1.808888 \\ \mathrm{H} & -0.565929 & 0.824122 & 2.011847 \\ \mathrm{H} & 3.335908 & -0.229132 & 0.780068 \\ \mathrm{H} & 1.830475 & 0.082563 & 2.963876 \\ \mathrm{H} & 2.167903 & 1.125786 & 1.081934 \\ \mathrm{H} & -0.579740 & -0.835069 & -0.414751 \\ \mathrm{H} & -0.458502 & 0.907497 & -0.420466 \\ \mathrm{H} & -0.448417 & -0.918604 & 1.974488 \\ \mathrm{H} & 3.009939 & -0.207231 & -1.887926 \\ \mathrm{H} & 1.570205 & -1.190909 & -2.236032 \\ \mathrm{H} & 1.491873 & 0.547330 & -2.447496\end{array}$

\section{TS13/14}

$\begin{array}{lrrr}\mathrm{C} & -0.602400 & -0.252941 & -0.507391 \\ \mathrm{C} & -0.671191 & -0.048685 & 1.017502 \\ \mathrm{C} & 0.780111 & -0.096319 & -0.902023 \\ \mathrm{C} & -1.649441 & -0.875595 & -1.283084 \\ \mathrm{H} & -1.035051 & 0.679580 & -1.078974 \\ \mathrm{H} & -1.599940 & 0.426982 & 1.341401 \\ \mathrm{H} & 1.143387 & -0.258249 & -1.914426 \\ \mathrm{H} & -2.653685 & -0.923829 & -0.873511 \\ \mathrm{H} & -1.498802 & -1.143888 & -2.325599 \\ \mathrm{C} & 0.593982 & 0.778786 & 1.325745 \\ \mathrm{C} & 1.609199 & 0.360013 & 0.231397 \\ \mathrm{H} & 0.972046 & 0.602443 & 2.334166 \\ \mathrm{H} & 2.341967 & 1.126134 & -0.050446 \\ \mathrm{H} & 2.214270 & -0.509228 & 0.558106 \\ \mathrm{H} & 0.380457 & 1.849194 & 1.234076 \\ \mathrm{H} & -0.626213 & -1.040699 & 1.482339\end{array}$

\section{TS13/16}

$\begin{array}{lrrr}\mathrm{C} & -0.986047 & 0.723425 & -1.196388 \\ \mathrm{C} & -0.922707 & 0.369150 & 0.530540 \\ \mathrm{C} & 0.336095 & 0.658259 & 1.285278 \\ \mathrm{C} & 1.417808 & -0.057254 & 0.406970 \\ \mathrm{C} & 0.661867 & -1.167885 & -0.370908 \\ \mathrm{C} & -0.778077 & -0.645100 & -0.499790 \\ \mathrm{H} & -1.285450 & 1.441486 & -0.223106 \\ \mathrm{H} & -1.584080 & -1.346703 & -0.708389 \\ \mathrm{H} & 0.628688 & -2.101286 & 0.199840 \\ \mathrm{H} & 1.865941 & 0.669439 & -0.277976 \\ \mathrm{H} & 0.214413 & 0.151465 & 2.253228 \\ \mathrm{H} & -0.127895 & 1.230207 & -1.634510 \\ \mathrm{H} & -1.940821 & 0.803393 & -1.715824 \\ \mathrm{H} & 1.109434 & -1.391940 & -1.343022 \\ \mathrm{H} & 2.222117 & -0.454682 & 1.029818 \\ \mathrm{H} & 0.524019 & 1.715052 & 1.485741\end{array}$

